\title{
Effects of early rehabilitation nursing on the treatment compliance of and therapeutic effect to elderly patients with fracture of the lower limbs.
}

\author{
Wan-Hong Luo ${ }^{1 \#, ~ Y i n-X u ~ W a n g ~}{ }^{1 \#, ~ J i a n-R o n g ~ W u}{ }^{2}$, Xiao-Juan Wang ${ }^{3}$, Xue-Ming Jing ${ }^{*}$, Jian-Ying \\ Jing 4 \\ ${ }^{1}$ Department of Rehabilitation Medicine, Affiliated Hospital of North Sichuan Medical College, Nanchong, Sichuan, \\ PR China \\ ${ }^{2}$ Outpatient Department of Stomatology, Affiliated Hospital of North Sichuan Medical College, Nanchong, Sichuan, \\ PR China \\ ${ }^{3}$ Department of Nutrition, Affiliated Hospital of North Sichuan Medical College, Nanchong, Sichuan, PR China \\ ${ }^{4}$ Department of Urology, Affiliated Hospital of North Sichuan Medical College, Nanchong, Sichuan, PR China \\ \#These authors contributed equally to this paper
}

\begin{abstract}
Objective: This study aims to discuss the effects of early rehabilitation nursing on the treatment compliance of and therapeutic effect to elderly patients with fracture of the lower limbs.

Methods: A total of 140 elderly patients with fracture of the lower limbs admitted to our hospital from March 2015 to June 2016 were divided into the control group $(n=70)$ and intervention group $(n=70)$ by the ball-touching method. The control group was treated by conventional nursing, and the intervention group was provided with early rehabilitation nursing. Treatment compliance, HSS scores, Harris scores, and complications were observed and compared.

Results: The control group has significantly lower treatment compliance than the intervention group $(\mathbf{p}<0.05)$. The intervention group has significantly higher HSS and Harris scores at 3, 6, and 12 months after the operation than the control group $(\mathbf{p}<\mathbf{0 . 0 5})$. The control group has significantly higher occurrence rate of complications than the control group $(\mathbf{p}<0.05)$.

Conclusions: Early rehabilitation nursing plays an important role in the treatment compliance of and therapeutic effect to elderly patients with fracture of the lower limbs. Early rehabilitation nursing improved the knee and hip joint functions effectively and reduced the occurrence rate of complications. Early rehabilitation nursing is worthy of clinical reference and application.
\end{abstract}

Keywords: Elderly patients with fracture of the lower limbs, Treatment compliance, Therapeutic effect, Early rehabilitation nursing.

Accepted on December 26, 2017

\section{Introduction}

Fracture of the lower limbs of elderly patients is a common clinical disease in the orthopedics department [1]. Morbidity increases yearly with the gradually intensifying aging trend. The elderly easily suffers from fracture of the lower limbs upon mild external forces or falls and rapid twisting of the lower limbs in daily life because of osteoporosis and hip muscle regression [2,3]. Elderly patients with fracture of the lower limbs have to lie in bed for a long time, which leads to many complications, such as pulmonary infection, thrombus of the lower extremity veins, bedsores, and uroschesis [4]. These complications may seriously influence fracture therapy and rehabilitation effect. Increasing treatment compliance and reducing postoperative complications are the keys to current nursing practice. In this study, a total of 140 elderly patients with fracture of the lower limbs were selected as the research objects. Results are introduced in the subsequent sections.

\section{Information and Methods}

\section{General information}

A total of 140 elderly patients with fracture of the lower limbs admitted to our hospital from March 2015 to June 2016 were selected and divided into the control group $(n=70)$ and intervention group $(n=70)$ by the ball-touching method. The control group had 40 males and 30 females aged 61-88 y (72.5 \pm 4.6 on average). With respect to fracture type, 28 cases were 
close fractures and 42 cases were open fractures. With regard to the cause of the fracture, 36 cases were caused by traffic accidents, 21 cases were caused by falls, and 13 cases were caused by overuse injuries. The intervention group had 41 males and 29 females aged $62-87$ y $(73.1 \pm 4.7$ on average). With respect to fracture type, 27 cases were close fractures and 43 cases were open fractures. With regard to the cause of the fracture, 38 cases were caused by traffic accidents, 20 cases were caused by falls, and 12 cases were caused by overuse injuries. The two groups exhibited no significant differences in terms of general information $(p>0.05)$, indicating that they are comparable.

\section{Methods}

The control group was treated by conventional nursing: Nurses should follow the instructions of the attending doctor strictly to introduce basic knowledge and key concepts of the disease, provide diet intervention, medication instructions, and conventional rehabilitation nursing, keep the patients in bed for the first three days after the operation, instruct patients to make on-bed and off-bed activities according to the disease and physical conditions, and provide discharge instructions.

The intervention group was provided with early rehabilitation nursing: (1) Limb function rehabilitation training: Nurses trained patients' limbs through on-bed activities after $3 \mathrm{~d}$ of the operation and assisted them in exercising their knee and hip joints passively through buckling and abduction. Moreover, nurses trained patients in exercising their quadriceps femoris, ankle joint, and gluteus positively. At $4 \mathrm{~d}$ to $7 \mathrm{~d}$ after the operation, nurses guided patients in exercising their knee and hip joints and upper limb muscles through positive flexion and abduction. At $8 \mathrm{~d}$ after the operation, nurses assisted patients in fowler position exercises and sitting training. Moreover, nurses assisted patients in flexion, adduction, and abduction training of the hip joints and in the gradual transitioning to standing and walking training. After the early rehabilitation training, activity intensity and complexity were increased gradually. (2) Skin nursing intervention and instruction on pressure sore prevention: An air bed was given to patients with high risks of pressure sores after traction. Nurses assisted patients to turn over every $2 \mathrm{~h}$, embrocate $50 \%$ safflower alcohol at the bony prominence position, massage appropriately, and keep the perineum clean to prevent poor stimulus of urination. (3) Psychological intervention: The psychological state of the patient was evaluated. Nurses listened to patients carefully and patiently, formulated an individualized nursing program, pacified unhealthy emotions (e.g., anxiety and agitation), asked patients to keep a peaceful and stable mind and psychological state, introduced several cases with good rehabilitation effect, boosted the patients' confidence in defeating the disease, encouraged the patients to cooperate with doctors and nurses, and increased the treatment compliance. (4) Pulmonary, urinary tract, and mouth care intervention: Patients were instructed to rinse their mouth or drink water immediately after eating to eliminate residual foods in the mouth. Nurses patted the back of the patients regularly and guided them to effectively take deep breaths and create productive coughs, which discharged secreta in the respiratory tract and prevented respiratory tract infection. Female patients kept their perineum dry and clean (i.e., by washing twice every day), increase water intake, and not hold urine.

\section{Observation indices}

Evaluation standards of treatment compliance: Complete compliance meant the patients followed the doctor's advice strictly and stick to standard treatment. Incomplete compliance meant the patients followed the doctor's advice basically and underwent some nonstandard treatments occasionally. Poor compliance means the patients rejected the doctor's advice completely and interrupted the treatment. Compliance $=($ complete compliance + incomplete compliance $)$ total cases $\times 100 \%$.

Function assessment of knee and hip joints: HSS scores of knee function and Harris scores of hip joints at 3, 6, and 12 months after the operation were used to evaluate the rehabilitation effect of knee and hip functions. High scores represent good function rehabilitation.

Complications: Occurrences of complications, including pulmonary infection, uroschesis, Deep Vein Thrombosis (DVT), and bedsores, in the two groups were observed in the follow-up visits.

\section{Statistical methods}

All relevant data were analysed by SPSS 22.0. HSS and Harris scores were expressed as $\overline{\mathrm{x}} \pm \mathrm{s}$, and the intergroup comparison was examined by t-test. Treatment compliance and occurrence rate of complications were expressed in percent, and the intergroup comparison was examined by $\chi^{2}$ test. $\mathrm{p}<0.05$ indicated statistically significant differences between the two groups.

\section{Results}

\section{Treatment compliance}

The control group had significantly lower treatment compliance than the intervention group $(\mathrm{p}<0.05)$. The results are shown in Table 1.

\section{HSS scores at different postoperative periods}

The intervention group had significantly higher HSS scores at 3,6 , and 12 months after the operation than the control group $(\mathrm{p}<0.05)$. The results are shown in Table 2 .

\section{Harris scores at different postoperative periods}

The intervention group had significantly higher Harris scores at 3,6 , and 12 months after the operation than the control group $(p<0.05)$. The results are shown in Table 3 . 
Effects of early rehabilitation nursing on the treatment compliance of and therapeutic effect to elderly patients with fracture of the lower limbs

\section{Occurrence rate of postoperative complications}

The control group had significantly higher occurrence rate of complications than the control group $(\mathrm{p}<0.05)$. The results are shown in Table 4.

Table 1. Comparison of compliance between the two groups ( $n(\%))$.

\begin{tabular}{lllll}
\hline Groups & $\begin{array}{l}\text { Complete } \\
\text { compliance }\end{array}$ & $\begin{array}{l}\text { Incomplete } \\
\text { compliance }\end{array}$ & $\begin{array}{l}\text { Poor } \\
\text { compliance }\end{array}$ & Compliance \\
\hline $\begin{array}{l}\text { Control } \\
(n=70)\end{array}$ & $35(50.0)$ & $22(31.4)$ & $13(18.6)$ & $57(81.4)$ \\
\hline $\begin{array}{l}\text { Intervention } \\
(n=70)\end{array}$ & $39(55.7)$ & $27(38.6)$ & $4(5.7)$ & $66(94.3)$ \\
\hline$X^{2}$ & & & 5.4232 \\
\hline$P$ & & & 0.0198 \\
\hline
\end{tabular}

Table 2. Comparison of HSS scores at different postoperative periods between the two groups $(\bar{x} \pm s$, score).

\begin{tabular}{lll}
\hline Groups & $\begin{array}{l}3 \text { months after } 6 \text { months after } 12 \text { months after } \\
\text { the operation }\end{array}$ & $\begin{array}{l}12 \text { operation } \\
\text { the operation }\end{array}$ \\
\hline
\end{tabular}

\begin{tabular}{llll}
\hline Control $(\mathrm{n}=70)$ & $80.4 \pm 7.3$ & $84.6 \pm 5.5$ & $88.7 \pm 8.3$ \\
\hline $\begin{array}{l}\text { Intervention } \\
(\mathrm{n}=70)\end{array}$ & $89.6 \pm 7.1$ & $92.5 \pm 7.4$ & $98.3 \pm 9.7$ \\
\hline $\mathrm{t}$ & 7.5587 & 7.1687 & 6.2914 \\
\hline $\mathrm{P}$ & 0.0000 & 0.0000 & 0.0000 \\
\hline
\end{tabular}

Table 3. Comparison of Harris scores at different postoperative periods between the two groups ( $\bar{x} \pm s$, score).

\begin{tabular}{llll}
\hline Groups & $\begin{array}{l}3 \text { months after } \\
\text { the operation }\end{array}$ & $\begin{array}{l}\mathbf{6} \text { months after } \\
\text { the operation }\end{array}$ & $\begin{array}{l}\mathbf{1 2} \text { months after } \\
\text { the operation }\end{array}$ \\
\hline Control $(n=70)$ & $51.7 \pm 6.4$ & $54.7 \pm 8.2$ & $57.4 \pm 6.3$ \\
\hline $\begin{array}{l}\text { Intervention } \\
(n=70)\end{array}$ & $64.6 \pm 5.5$ & $68.9 \pm 7.1$ & $73.5 \pm 6.2$ \\
\hline$t$ & 12.7899 & 13.2672 & 15.2393 \\
\hline$P$ & 0.0000 & 0.0000 & 0.0000 \\
\hline
\end{tabular}

Table 4. Comparison of occurrence rate of complications between the two groups (n (\%)).

\begin{tabular}{llllll}
\hline Groups & Pulmonary infection & DVT & Bedsore & Uroschesis & Complications \\
\hline Control $(\mathrm{n}=70)$ & $5(7.1)$ & $2(2.9)$ & $1(1.4)$ & $2(2.9)$ & $10(14.3)$ \\
\hline Intervention $(\mathrm{n}=70)$ & $2(2.9)$ & $1(1.4)$ & $0(0.0)$ & $0(0.0)$ & $3(4.3)$ \\
\hline $\mathrm{X}^{2}$ & & & & 4.1551 \\
\hline $\mathrm{P}$ & & & & 0.0415 \\
\hline
\end{tabular}

\section{Discussion}

Fracture of the lower limbs is a common fracture type in the elderly group. Fall accidents from high places, accidental falls, and traffic accidents will all cause fracture of the lower limbs [5]. Although conventional therapy, such as internal fixation with intramedullary nails, can achieve good clinical effect, elderly patients easily suffer from poor outcomes, such as nonunion and ischemic necrosis of the bones, because of functional recessions and limited body tolerance, which may influence postoperative recovery and rehabilitation effect significantly [6]. These outcomes should be the focus of attention in clinics. Elderly patients with fracture of the lower limbs require traction and often have to lie on bed for a long time [7]. Under this circumstance, the venous return slows down significantly, thereby causing circulatory disturbance. Moreover, the elderly group easily suffers from complications (e.g., lower limb DVT) because of poor blood vessel power and elasticity [8].

Traditionally, elderly patients with fracture of the lower limbs are advised to lie in bed after internal fixation operation, but are not advised to undergo function rehabilitation training. Recently, continuous clinical studies and practices indicated that function rehabilitation training as early as possible after the operation can lead to an outstanding effect [9]. Specifically, the important role of early rehabilitation nursing in increasing the treatment compliance of patients, reducing postoperative complications, and improving quality of life and prognosis is highly approved. Early rehabilitation nursing intervention mainly includes limb function rehabilitation training, pulmonary, urinary tract, mouth, skin, and sore care, and psychological intervention. Early rehabilitation nursing intervention can reduce the occurrence rate of complication, accelerate fracture union, and alleviate the unhealthy emotions of patients with fracture of the lower limbs significantly, thereby encouraging them to handle the disease with confidence and keep a calm attitude toward the rehabilitation process [10]. Moreover, early rehabilitation nursing intervention can increase the treatment compliance of patients and accelerate disease recovery. Meanwhile, early rehabilitation nursing of elderly patients with fracture of the lower limbs can do the following: improve the individualized rehabilitation nursing program after comprehensive and systematic evaluation of the basic conditions of patients; solve the nursing problem progressively; enhance the emotional communication between nurses and patients; correct and handle potential problems in a timely manner; shorten the length of stay and treatment time; and increase the nursing 
satisfaction of patients, thereby improving nursing quality significantly.

\section{Conclusion}

In this study, the intervention group has significantly higher HSS and Harris scores at 3, 6, and 12 months after the operation than the control group $(p<0.05)$. The control group has significantly higher occurrence rate of complications than the control group $(\mathrm{p}<0.05)$. Early rehabilitation nursing plays an important role in the treatment compliance and in the therapeutic effect on elderly patients with fracture of the lower limbs. Early rehabilitation nursing can improve the knee and hip joint functions effectively and reduce the occurrence rate of complications. Early rehabilitation nursing is worthy of clinical reference and application.

\section{References}

1. Frouzan A, Masoumi K, Delirroyfard A, Mazdaie B, Bagherzadegan E. Diagnostic accuracy of ultrasound in upper and lower extremity long bone fractures of emergency department trauma patients. Electr Phys 2017; 9: 5092-5097.

2. Tins BJ, Garton M, Cassar-Pullicino VN, Tyrrell PNM, Lalam R, Singh J. Stress fracture of the pelvis and lower limbs including atypical femoral fractures-a review. Insig Imag 2015; 6: 97-110.

3. Zhang J, Ma J. The influence of dextro-thyroxine (Dthyroxine) on the metabolic elimination of anesthetic agent propofol. Lat Am J Pharm 2017; 36: 2329-2332.

4. Zatta D, Perassolo MS. Adherence promotion to pharmacological treatment in patients with type 2 diabetes mellitus in a pharmacy in Southern Brazil. Lat Am J Pharm 2017; 36: 2315-2323.

5. Bozorgi F, Azar MS, Montazer SH, Chabra A, Heidari SF, Khalilian A. Ability of ultrasonography in detection of different extremity bone fractures; a case series study. Emerg (Tehran) 2017; 5: 15.

6. Joeris A, Lutz N, Blumenthal A, Slongo T, Audigé L. The AO pediatric comprehensive classification of long bone fractures (PCCF). Acta Orthop 2017; 88: 129-132.

7. Chandrakar C, Sharma M. Approach for design of early warning monitoring system for detection of the abnormal cardiac behaviour of any individual. Biomed Res India 2017; 28: 81-86.

8. Creasy KR, Lutz BJ, Young ME, Stacciarini JMR. Clinical implications of family-centered care in stroke rehabilitation. Rehabil Nurs 2015; 40: 349-359.

9. Amr AEGE, Abdalla MM. Anticancer activities of some synthesized 2, 4, 6-trisubstituted pyridine candidates. Biomed Res India 2016; 27: 731-736.

10. Spasser MA, Weismantel A. Mapping the literature of rehabilitation nursing. J Med Libr Assoc 2006; 94: 137-142.

\section{*Correspondence to}

Xue-Ming Jing

Department of Urology

Affiliated Hospital of North Sichuan Medical College

PR China 